\title{
Interventional Procedures in Episodic Migraine
}

\author{
Brian M. Plato ${ }^{1}$ D $\cdot$ Mandy Whitt ${ }^{1}$
}

Accepted: 28 October 2020 / Published online: 12 November 2020

(C) Springer Science+Business Media, LLC, part of Springer Nature 2020

\begin{abstract}
Purpose of Review Migraine is a common disorder which in many cases can be adequately treated with medications. However, there are some patients who may either not respond to medications or have contraindications to their use. In this review, we will evaluate the available literature on the interventional procedures available to treat patients with episodic migraine. We will review the technical details of performing the procedures, the potential mechanisms of action, and available data on their effectiveness. Recent Findings Recent studies conducted on the subject of interventional procedures including peripheral nerve blocks and onabotulinumtoxinA indicate that some patients with episodic migraine may find benefit from such procedures. Summary In patients with episodic migraine whom traditional treatments have not been effective or are contraindicated, interventional procedures may offer an opportunity to provide additional treatment options.
\end{abstract}

Keywords Episodic migraine $\cdot$ Procedures $\cdot$ Nerve blocks $\cdot$ OnabotulinumtoxinA

\section{Introduction}

Migraine is a primary headache disorder with clearly defined diagnostic characteristics established within the International Classification of Headache Disorders, third edition [1]. The diagnostic criteria of migraine include attacks consisting of both headache features as well as non-headache features. Migraine is a common disorder, affecting $12 \%$ of the population in the USA [2] and is responsible for 5 to 9 million visits with primary care annually in the USA $[3,4]$. According to ICHD-3 criteria, patients with migraine can then be further diagnosed with chronic migraine $(\mathrm{CM})$ when having 15 or more headache days per month with at least 8 days meeting ICHD criteria for migraine with or without aura [1]. Patients can also be diagnosed with status migrainosus (SM) when having a migraine attack lasting more than $72 \mathrm{~h}$ causing disability [1]. While there are no specific diagnostic criteria for episodic migraine (EM) in ICHD-3, the term refers to those individuals with fewer than 15 headache days per month.

This article is part of the Topical Collection on Episodic Migraine

Brian M. Plato

brian.plato@gmail.com

1 Norton Neuroscience Institute, Louisville, KY, USA
Those individuals with CM may result in having migraines that can be a more severe and longer-lasting, more difficult treat and have greater functional impairment than those with EM [5]. To further differentiate patients, those with EM can be further subclassified as having either lowfrequency episodic migraine (LFEM) or high-frequency episodic migraine (HFEM). There is no clear standardization of the definition of LFEM versus HFEM, but generally ranges between 8 to 14 or 10 to 14 headache days per month are used to characterize HFEM [6, 7].

Despite the numerous recent advances in the pharmacological and neuromodulatory options for treating migraine, there remain numerous reasons why these treatments may not be the best available options for patients. There may be concomitant conditions such as cardiovascular or cerebrovascular diseases, renal or hepatic impairment, pregnancy, psychiatric comorbidities, or drug interactions where these available treatments may not be the ideal options. Due to the more refractory nature of CM, many studies involving interventional procedures for treating migraine focus more on the population of patients with CM than those with EM.

This review aims to evaluate interventional procedures that are utilized in clinical practice and explore the physiological rationale for their use, the techniques involved in performing the procedures, and the available evidence for their benefit to patients with EM. 


\section{Peripheral Nerve Blocks in Episodic Migraine}

Peripheral nerve blocks (PNBs) are commonly used procedures as they are generally thought of as safe and welltolerated procedures that in the outpatient setting can provide relief within minutes to patients of not only of a headache but also of allodynia and photophobia [8]. The pain modulating effects of PNBs can last in some patients up to several months, which far outlasts the pharmacokinetic profile of the type of local anesthetic that is utilized in the procedure [9]. The analgesic effects of PNBs are not fully understood, but pain suppression is likely due to the selective blocking of sensory fibers while sparing motor functions then producing central pain modulating effects via second-order neurons in the trigeminocervical complex [10•].

Most commonly, PNBs are performed in the anatomical distribution of the pain that the patient is experiencing, although due to the convergence of numerous nociceptive systems centrally, there may be analgesic effects outside of the distribution of the nerve anesthetized.

\section{Greater Occipital Nerve}

Perhaps one of the more commonly injected peripheral nerves in EM is the greater occipital nerve (GON). The GON is a branch of $\mathrm{C} 2$ which innervates the posterior scalp medially from the occipital protuberance to the vertex. While there can be anatomical variation between patients, oftentimes the GON is located adjacent to the occipital artery, which can many times be palpated or is approximately $2 \mathrm{~cm}$ lateral to the occipital protuberance.

The technique for GON block includes the injection of solution over the distribution of the GON. There has been some lack of consensus of what solution to use in the preparation of GON block. Many practitioners will use lidocaine 1$2 \%$ (quicker onset with shorter duration), bupivacaine 0.25 $0.5 \%$ (delayed onset with longer duration), or a combination of the two. They may also use the combination of local anesthetic with a corticosteroid; however, a randomized, doubleblind control study for the benefit of the addition of corticosteroid to the local anesthetic found no benefit over local anesthetic alone [11].

\section{Lesser Occipital Nerve}

The lesser occipital nerve (LON) is a branch of $\mathrm{C} 2$ and $\mathrm{C} 3$ innervating the posterior scalp laterally down to the upper neck. The LON can be injected approximately $1-2 \mathrm{~cm}$ medially to the mastoid process. Similarly to GON block, a mixture of lidocaine and bupivacaine is most commonly used for injection. Blockade of the LON has not been systematically studied in EM but is commonly included with blockade of the GON and other PNBs.

\section{Auriculotemporal Nerve}

The auriculotemporal nerve (ATN) is a branch of V3 which innervates above the ear and temporalis muscle. Similar to other PNBs, blockade of the ATN can be achieved with injection of local anesthetic just anterior to the tragus of the ear. Patients will experience anesthesia anterior and superior to the ear if this nerve is injected properly. Similarly to LON blocks, blockade of the ATN has not been systematically reviewed.

\section{Supraorbital and Supratrochlear Nerves}

The supraorbital nerve (SON) and supratrochlear nerve (STN) are both branches of $\mathrm{V} 1$ providing sensation above the eyebrow medially (STN) and laterally (SON). Injection of the STN is performed at or slightly above the level of the eyebrow over its medial border. The injection of the SON is performed approximately $2 \mathrm{~cm}$ lateral to the SON injection. For both injections, local anesthetic is injected in the identified locations. Corticosteroids should be avoided in these locations due to the risk of a cosmetically viewable area of lipoatrophy that can occur with intramuscular steroid injections.

Blockades of the SON and STN are commonly performed with other PNBs. A comment in the author's experience is that, many times, patients will find these particular PNBs to be the most tender of the locations of nerve blocks during the injection itself.

The effectiveness of peripheral nerve blocks has been reviewed in a variety of studies that have significant variability between them, including populations of patients (EM and/ or $\mathrm{CM}$ ), preventive or acute treatment, outcome measures, the peripheral nerves blocked, and medications administered. A limitation to conducting placebo-controlled studies with anesthetics is that patients will be aware of whether they experience the sensory effects of the anesthetic or not. Unfortunately, there are few studies specifically evaluating patients with EM, rather than the more frequently studied patient population with $\mathrm{CM}$.

Reviewing more recent studies that specifically include patients with EM, a randomized, double-blinded study used as an active intervention $2.5 \mathrm{~mL}$ bupivacaine $0.5 \%$ plus $20 \mathrm{mg}$ methylprednisolone versus a placebo solution of $0.25 \mathrm{~mL}$ lidocaine $1 \%$ for prevention of migraine. In patients with episodic migraine in the 28 -day period post-injection, there was no difference in frequency of migraine compared with placebo in the patients who received GON blockade [12].

With the combined treatment of GON and SON blockade in a population of patients with episodic migraine, statistically 
significant reductions in both headache frequency as well as headache severity were seen compared with placebo. Patients with episodic migraine treated with combined GON and SON blocks experienced a reduction from 4.9 headache days per month down to 2.3 (a $53.1 \%$ reduction), compared with placebo experiencing only a reduction from 5.2 headache days per month down to 4.4 (a $15.1 \%$ reduction) [13].

For the acute treatment of EM, a recent retrospective review of patients is reported as having EM presenting with acute migraine received GON blocks at the time of their office visits. Nearly all patients $(99.3 \%)$ received local anesthetic combined with corticosteroids. Using self-reported pain improvement scores, $82 \%$ of patients reported their pain response to GON block as moderate or significant [14].

A randomized controlled study for treatment of acute migraine in the emergency room setting showed GON block as effective as the combination of IV dexketoprofen and metoclopramide, and both were more beneficial than placebo. This study showed patients had a $44.4 \%$ decrease on the pain scale score for headache with a GON block of a solution of $1 \mathrm{~mL} 0.5 \%$ bupivacaine and $1 \mathrm{~mL}$ normal saline vs. a placebo injection of $2 \mathrm{~L}$ of normal saline [15].

In conclusion, it appears that for EM, PNBs can be helpful preventively when performed as combined GON and SON blocks and for acute migraine as a GON block.

\section{Sphenopalatine Ganglion}

The sphenopalatine ganglion (SPG) is a region of neurons that are housed within the pterygopalatine fossa containing sensory fibers that innervate V2 as well as parasympathetic and sympathetic fibers. Blockade of the SPG has long been utilized in treating headache disorders, perhaps most commonly in cluster headache; however, there have been several studies in migraine, most commonly for the acute treatment of migraine.

The blockade of the SPG can be achieved by several methods which range from rather invasive transcutaneous or intraoral injection to less invasive methods. The less invasive methods of SPG blockade are aimed at instilling a local anesthetic to the mucosa of the lateral posterior wall of the nasal cavity. This is one PNB that patients may be able to perform on their own as needed at home. One method is for patients to use a syringe filled with lidocaine that is inserted to the tip of the nostril and lidocaine is slowly introduced to the posterior portion of the nasal cavity. An additional method is to use a cotton-tipped applicator saturated with lidocaine that is inserted transnasally to the posterior wall of the nasal cavity. Flexible catheters for SPG blockade have been available for practitioners to perform the procedure in a minimally invasive manner in the office.
The effectiveness of SPG blockade in migraine has been most studied for the acute treatment of migraine. A placebocontrolled trial for intranasal lidocaine demonstrated that patients treated with lidocaine were more likely to experience headache relief within $15 \mathrm{~min}$ as opposed to those who received placebo; however, many experienced recurrence of headache soon after experiencing initial relief [16]. At a follow-up study of patients treated at home with intranasal lidocaine, $35.8 \%$ of patients experienced headache relief within 15 min when treated with $4 \%$ lidocaine, compared with $7.4 \%$ of patients receiving placebo [17]. Another recent uncontrolled retrospective study had $70.9 \%$ of patients with headache freedom at $15 \mathrm{~min}$ with a single treatment of transnasal SPG block of $2 \mathrm{~mL}$ of $2 \%$ lidocaine in each nostril [18].

A transcutaneous approach to treat status migrainosus has recently been studied with patients undergoing a suprazygomatic SPG block with a $5 \mathrm{~mL}$ mixture of dexamethasone $(1 \mathrm{~mL})$ and $0.5 \%$ ropivacaine $(4 \mathrm{~mL})$. Patients reported a $67.2 \%$ reduction in pain severity $30 \mathrm{~min}$ following the procedure, and this improvement of pain was seen after failure of other medical treatment for acute migraine [19].

In conclusion, for $\mathrm{EM}$, there is not any available literature on the preventive effects of SPG blockade; however, there is some evidence for using SPG blocks for the acute treatment of migraine, particularly when this can be taught to patients how to perform at home.

A comment from the author is that, at the time of preparation of this manuscript, the COVID-19 pandemic was spreading, and our practice had discontinued the offering of intranasal-based procedures for the acute treatment of migraine performed in the office.

\section{Trigger Point Injections}

Trigger point injections (TPIs) are commonly performed procedures for a variety of conditions that include musculoskeletal pain. Trigger points are a finding on physical examination as a sign of myofascial pain and inflammation. Trigger points have been identified as present in $94 \%$ of patients with migraine compared to $29 \%$ of controls, with the number of trigger points related to increased attack frequency. The majority of these trigger points were found in the temporal and suboccipital regions [20].

TPIs are performed by palpating an area of tenderness reported by the patient. After palpation, there may be more erythema at the site of tenderness, reaffirming underlying inflammation of the muscles. A small quantity $(0.1-0.25 \mathrm{~mL})$ of a local anesthetic such as lidocaine or bupivacaine is then injected into the site of tenderness.

There are minimal studies using TPIs in EM; however, one prospective study found that combining GON blocks with 
TPIs for acute pain resulted in $89.5 \%$ of patients seeing headache improvement $20 \mathrm{~min}$ after injection [21]. A second study of patients with both EM and CM receiving weekly injections for a period of 12 weeks found that $59.6 \%$ of patients reported being "much or very much improved" after the 12 -week treatment period [22].

In the authors' experience, TPIs may be helpful when performing PNBs when patients complain of coexistent neck and shoulder pain associated with acute migraine.

\section{OnabotulinumtoxinA}

Botulinum toxin has been used for conditions associated with excessive muscular contraction since the 1980s and has been found to have pain-relieving properties in some conditions. In 2010, the United States Food and Drug Administration approved the preparation of botulinum toxin, onabotulinumtoxinA (brand name Botox), for the prevention of chronic migraine based upon two large trials [23, 24]. Previous analysis of available data for the use of onabotulinumtoxinA in patients with EM has failed to provide adequate support for its use in patients with episodic migraine [25].

Recognizing that the disability associated with HFEM is similar to that seen in $\mathrm{CM}$, a recent prospective study analyzed both the early and later benefits of onabotulinumtoxinA in both patient populations [26•]. For the purposes of this study, HFEM was defined as patients with $8-14$ headache days per month. Patients in both groups were treated similarly with quarterly injections using the PREEMPT injection protocol. The response to onabotulinumtoxinA was evaluated at the times points of $6,12,18$, and 24 months from baseline. Evaluations were made comparing reductions in headache and migraine days per month, headache intensity improvements, the reduction in acute medication use, and measures of impact and disability.

At 6 months, patients with HFEM had a reduction in headache days per month from 13.4 down to 9.4 , with a $34.2 \%$ reduction in the number of migraine days per month. In terms of analgesic use, patients with HFEM had a reduction from 21.4 pills per month down to 14.4. After a period of another 6 months (12 months from baseline), patients with HFEM had a further slight reduction to a total of 8.7 headache days per month.

Comparing HFEM to CM, patients had similar reductions in headache frequency ( $44.7 \%$ for CM and $41.2 \%$ for HFEM) as well as similar reductions in pain intensity, analgesic use, preventive medication use, and MIDAS scores.

Based upon this recent single non-blinded study, patients with HFEM (8-14 headache days per month) who have not had adequate responses to other interventions may be appropriate patients to consider using onabotulinumtoxinA for preventive treatment.

\section{Summary}

For patients with EM, there are situations where treatment with oral medication is at times contraindicated due to other comorbidities or fails to provide complete and adequate responses. As a result, interventional procedures may provide an option for treatment in these situations. Unfortunately, many of these treatments do not have adequate studies to support their use, particularly to the degree that regulatory bodies and insurers would permit. Based upon the reviewed literature above, there certainly are some encouraging results that might be applicable to clinical practice and result in future research projects.

\section{Compliance with Ethical Standards}

Conflict of Interest The authors declare that they have no conflicts of interest.

Human and Animal Rights and Informed Consent This article does not contain any studies with human or animal subjects performed by any of the authors.

\section{References}

Papers of particular interest, published recently, have been highlighted as:

- Of importance

•• Of major importance

1. Headache Classification Committee of the International Headache Society. The International Classification of Headache Disorders, 3rd edition (beta version). Cephalalgia. 2013;33:629-8008.

2. Lipton RB, Bigal ME, Diamond M, Freitag F, Reed ML, Stewart WF, et al. Migraine prevalence, disease burden, and the need for preventive therapy. Neurology. 2007;68(5):343-9.

3. Gibbs TS, Fleischer AB Jr, Feldman SR, Sam MC, O'Donovan CA. Health care utilization in patients with migraine: demographics and patterns of care in the ambulatory setting. Headache. 2003;43(4): $330-5$.

4. Collins JG. Prevalence of selected chronic conditions, United States, 1979-1981. Vital Health Stat. 1986;10:1-66.

5. Katsarava Z, Buse DC, Manack AN, Lipton RB. Defining the differences between episodic migraine and chronic migraine. Curr Pain Headache Rep. 2012;16(1):86-92.

6. Katsarava Z, Manack A, Yoon MS, Obermann M, Becker H, Dommes P, et al. Chronic migraine: classification and comparisons. Cephalalgia. 2011;31(5):520-9.

7. Torres-Ferrús M, Quintana M, Fernandez-Morales J, Alvarez-Sabin J, Pozo-Rosich P. When does chronic migraine strike? A clinical comparison of migraine according to the headache days suffered per month. Cephalalgia. 2017;37(2):104-13. 
8. Young W, Cook B, Malik S, Shaw J, Oshinsky M. The first 5 minutes after greater occipital nerve block. Headache. 2008;48(7): 1126-8.

9. Afridi SK, Shields KG, Bhola R, Goadsby PJ. Greater occipital nerve injection in primary headache syndromes - prolonged effects from a single injection. Pain. 2006;122:126-9.

10. Blumenfeld A, Ashkenazi A, Napchan U, et al. Expert consensus recommendations for the performance of peripheral nerve blocks for headaches - a narrative review. Headache. 2013;53:437-46 This review and series of recommendations are important in further understanding the mechanisms of how nerve blocks may work and the settings in which they may be utilized in treating patients with headache disorders.

11. Ashkenazi A, Matro R, Shaw JW, Abbas MA, Silberstein SD. Greater occipital nerve block using local anaesthetics alone or with triamcinolone for transformed migraine: a randomised comparative study. J Neurol Neurosurg Psychiatry. 2008;79:415-7.

12. Dilli E, Halker R, Vargas B, Hentz J, Radam T, Rogers R, et al. Occipital nerve block for the short-term preventive treatment of migraine: a randomized, double-blinded, placebo-controlled study. Cephalalgia. 2015;35(11):959-68.

13. Özer D, Bölük C, Börü ÜT, Altun D, Taşdemir M, Toksoy CK. Greater occipital and supraorbital nerve blockade for the preventive treatment of migraine: a single-blind, randomized, placebocontrolled study. Curr Med Res Opin. 2019;35(5):909-15.

14. Allen SM, Mookadam F, Cha SS, Freeman JA, Starling AJ, Mookadam M. Greater occipital nerve block for acute treatment of migraine headache: a large retrospective cohort study. J Am Board Fam Med. 2018;31(2):211-8.

15. Korucu O, Dagar S, Çorbacioglu ȘK, Emektar E, Cevik Y. The effectiveness of greater occipital nerve blockade in treating acute migraine-related headaches in emergency departments. Acta Neurol Scand. 2018;138:212-8.

16. Maizels M, Scott B, Cohen W, Chen W. Intranasal lidocaine for treatment of migraine: a randomized, double-blind, controlled trial. JAMA. 1996;276:319-21.

17. Morris Maizels MD, Geiger AM. Intranasal lidocaine for migraine: a randomized trial and open-label follow-up. Headache. 1999;39: 543-51.
18. Binfalah M, Alghawi E, Shosha E, Alhilly A, Bakhiet M. Sphenopalatine ganglion block for the treatment of acute migraine headache. Pain Res Treat. 2018;2516953.

19. Mehta D, Leary MC, Yacoub HA, el-Hunjul M, Kincaid H, Koss $\mathrm{V}$, et al. The effect of regional anesthetic sphenopalatine ganglion block on self-reported pain in patients with status migrainosus. Headache. 2019;59(1):69-76.

20. Calandre EP, Hidalgo J, Garcia-Leiva JM, Rico-Villademoros F. Trigger point evaluation in migraine patients: an indication of peripheral sensitization linked to migraine predisposition? Eur J Neurol. 2006;13:244-9.

21. Ashkenazi A, Young WB. The effects of greater occipital nerve block and trigger point injection on brush allodynia and pain in migraine. Headache. 2005;45:350-4.

22. Garcia-Leiva JM, Hidalgo J, Rico-Villademoros F, Moreno V, Calandre EP. Effectiveness of ropivacaine trigger points inactivation in the prophylactic management of patients with severe migraine. Pain Med. 2007;8:65-70.

23. Diener HC, Dodick DW, Aurora SK, Turkel CC, DeGryse RE, Lipton RB, et al. OnabotulinumtoxinA for treatment of chronic migraine: results from the double-blind, randomized, placebocontrolled phase of the PREEMPT 2 trial. Cephalalgia. 2010;30(7):804-14.

24. Aurora SK, Dodick DW, Turkel CC, DeGryse RE, Silberstein SD, Lipton RB, et al. PREEMPT 1 chronic migraine study group. OnabotulinumtoxinA for treatment of chronic migraine: results from the double-blind, randomized, placebo-controlled phase of the PREEMPT 1 trial. Cephalalgia. 2010;30(7):793-803.

25. Herd CP, Tomlinson CL, Rick C, et al. Botulinum toxins for the prevention of migraine in adults. Cochrane Database Syst Rev. 2018;6:CD011616.

26. Alpuente A, Gallardo VJ, Torres-Ferrus M, Alvarez-Sabin J, PozoRosich P. Early efficacy and late gain in chronic and highfrequency episodic migraine with onabotulinumtoxinA. Eur Adac of Neurol. 2019;26:1464-70 This study is important in exploring the belief that only patients with chronic migraine may find benefit from onabotulinumtoxin $A$.

Publisher's Note Springer Nature remains neutral with regard to jurisdictional claims in published maps and institutional affiliations. 